\title{
ARrió $\mid$ Vitimização e perpetração da violência por parceiro íntimo em idosos: estudo transversal, Florianópolis, Santa Catarina, 2013/2014*
}

doi: 10.1590/51679-49742021000400009

Victimization and perpetration of intimate partner violence among elderly people: a cross-sectional study, Florianópolis, Santa Catarina, Brazil, 2013/2014

Victimización y perpetración de violencia por pareja íntima en ancianos: estudio transversal, Florianópolis, Santa Catarina, Brasil, 2013/2014

\author{
Deise Warmling' - (D) orcid.org/0000-0001-9235-6271 \\ Thays Berger Conceição' - (1) orcid.org/0000-0002-9445-3427 \\ Sheila Rubia Lindner ${ }^{1}$ - (1) orcid.org/0000-0001-9724-1561 \\ Elza Berger Salema Coelho' - (1) orcid.org/0000-0001-7407-6786 \\ 'Universidade Federal de Santa Catarina, Departamento de Saúde Pública, Florianópolis, SC, Brasil
}

\begin{abstract}
Resumo
objetivo: Verificar a prevalência de violência por parceiro íntimo em idosos e identificar os fatores associados. Métodos: Estudo transversal de base populacional, oriundo do estudo EpiFloripa Idoso, com idosos residentes em Florianópolis, Santa Catarina, Brasil, entre 2013 e 2014. Foram descritas as prevalências e analisados os fatores associados por regressão de Poisson, para violência sofrida e perpetrada, estratificada por sexo. Resultados: Entre 651 idosos, $48,3 \%$ do sexo masculino e $46,4 \%$ do feminino sofreram violência por parceiro íntimo. A violência psicológica sofrida ( $48,3 \%$ e 44,8\%) e perpetrada ( $49,8 \%$ e 44,5\%) predominou nos sexos masculino e feminino, respectivamente. A violência perpetrada foi associada à autopercepção de saúde ruim/muito ruim $(\mathrm{RP}=1,74)$ para idosos homens, e à autopercepção de saúde regular $(\mathrm{RP}=1,53)$ e estado civil 'separada/divorciada' $(\mathrm{RP}=1,86)$ entre as idosas. Conclusão: Constatou-se uma simetria entre os sexos na prevalência de violência por parceiro íntimo, em todas as direcionalidades da violência analisadas.
\end{abstract}

Palavras-chave: Violência por Parceiro Íntimo; Prevalência; Idoso; Estudos Transversais.

\footnotetext{
*Artigo derivado de tese de doutorado acadêmico intitulada 'Violência por parceiro íntimo e qualidade de vida em idosos residentes em Florianópolis, Santa Catarina: estudo de base populacional', defendida por Deise Warmling junto ao Programa de Pós-Graduação em Saúde Coletiva da Universidade Federal de Santa Catarina, em 10 de julho de 2018. Estudo financiado com recursos do Conselho Nacional de Desenvolvimento Científico e Tecnológico (CNPq)/Ministério da Ciência, Tecnologia Comunicações e Inovações (MCTI): Processo n 569834/2008-2.
}

\section{Correspondência:}

Deise Warmling - Universidade Federal de Santa Catarina, Departamento de Saúde Pública, Campus Universitário, Rua Delfino Conti, S/N, Trindade, Florianópolis, SC, Brasil. CEP: 88040-370

E-mail: deisentr@gmail.com 


\section{Introdução}

A violência contra a pessoa idosa tende a ocorrer no ambiente doméstico, geralmente cometida por familiares, cuidadores, inclusive pelo parceiro. ${ }^{1} \mathrm{~A}$ violência perpetrada por parceiro íntimo define-se por atos de agressão física, abuso psicológico, comportamento controlador; e abuso econômico, identificável na relação íntima. ${ }^{2,3}$ Recorrente na literatura, essa forma e objeto de violência é abordada com ênfase da violência por parceiro íntimo contra mulheres na idade reprodutiva, ${ }^{4} 0$ homem investigado como agressor e a mulher como vítima, observando-se pouca ênfase na possibilidade de a violência ser cometida pela mulher. ${ }^{5}$

\section{Estar em situação de violência traz consequências sociais para os idosos, como baixa estima, isolamento social, medo e insegurança. Ainda há implicações na saúde física e mental, como maiores proporções de relatos de dores de cabeça e osteomusculares, ansiedade, estressee ideação suicida, ao se considerar a própria velhice sob uma perspectiva negativa.}

Segundo a Organização Mundial da Saúde, aproximadas $30 \%$ das mulheres no mundo sofreram algum tipo de violência por parceiro íntimo, enquanto um estudo de revisão sistemática, sobre publicações oriundas de países industrializados de língua inglesa, identificou prevalência de aproximadamente $20 \%$ em homens. ${ }^{6,7}$ Estudo de amostra de base populacional, realizado no Canadá, ao analisar a violência por parceiro íntimo em idosos, estratificada por sexo e direcionalidade da violência, encontrou proporções superiores de vitimização nos homens (4,9\%) quando comparados às mulheres $(3,3 \%){ }^{8}$

Destacam-se, como fatores associados à violência por parceiro íntimo sofrida entre idosos: uso de álcool, depressão, baixa renda, baixa escolaridade, idade entre 60 e 69 anos, ser divorciado ou separado, autoavaliação de saúde ruim ou péssima, comprometimento funcional, além de exposição pregressa à violência. ${ }^{8-10}$
Estar em situação de violência traz consequências sociais para os idosos, como baixa estima, isolamento social, medo e insegurança. ${ }^{11}$ Ainda há implicações na saúde física e mental, como maiores proporções de relatos de dores de cabeça e osteomusculares, ansiedade, estresse e ideação suicida, ao se considerar a própria velhice sob uma perspectiva negativa. ${ }^{12}$ Apesar do impacto à saúde em consequência da violência cometida por parceiro íntimo, em ambos os sexos, predomina a investigação da violência contra a mulher.

Os estudos sobre a violência contra 0 idoso têm se concentrado na investigação das vítimas de maus tratos, praticados, em geral, por familiares ou cuidadores. ${ }^{13} \mathrm{~A}$ violência por parceiro íntimo entre idosos é subavaliada. Ela pode ser incluída na definição geral de violência familiar e, nesse caso, impede-se um entendimento claro e detalhado do fenômeno.

Uma revisão sistemática sobre prevalência e fatores associados à violência por parceiro íntimo na população idosa, realizada a partir de estudos de base populacional, encontrou apenas quatro pesquisas conduzidas exclusivamente com idosos, uma vez que a maioria das publicações os analisam em conjunto com os adultos, ocultando as especificidades do grupo etário mais avançado. ${ }^{14}$ Considerando-se os impactos negativos da violência por parceiro íntimo ao longo da vida, inclusive no envelhecimento, evidencia-se a relevância de pesquisas de base populacional que investiguem tal desfecho entre idosos dos sexos masculino e feminino. É essencial, para a sociedade, assegurar a esse grupo etário condições de vida e saúde dignas, proporcionando-lhes um processo de envelhecimento seguro e saudável.

0 objetivo deste estudo foi verificar a prevalência de violência por parceiro íntimo em idosos e identificar seus fatores associados.

\section{Métodos}

Trata-se de um estudo transversal de base populacional e domiciliar, sobre dados coletados na segunda onda (2013/2014) do estudo longitudinal EpiFloripa Idoso, que investiga as condições de vida e saúde das pessoas idosas ( $\geq 60$ anos) residentes no município de Florianópolis.

Em 2009, Florianópolis, capital do estado de Santa Catarina, possuía população total de 421.240 habitantes, dos quais $49.793,11,5 \%$ da população, com idade 
igual ou superior a 60 anos. 0 índice de desenvolvimento humano municipal (IDH-M) era de $0,847 .^{15}$

A seleção da amostra da linha de base (2009/2010) ocorreu em dois estágios. 0 primeiro consistiu em 420 setores censitários urbanos selecionados, em ordem crescente, de acordo com a renda dos chefes de família. Foram sorteados, sistematicamente, 80 setores (oito para cada decil de renda), enquanto, no segundo estágio, as unidades de estudo foram os domicílios.

Para o EpiFloripa Idoso, o cálculo amostral foi realizado utilizando-se o programa Epi Info versão 6.04, dentro dos seguintes parâmetros: tamanho da população igual a 44.460 hab.; prevalência desconhecida de 50\%; erro amostral igual a 4 pontos percentuais; intervalo de confiança de $95 \%$; e efeito de delineamento igual a 2. Acrescentaram-se ao quantitativo calculado inicialmente $20 \%$ para perdas previstas e $15 \%$ para estudos de associação, totalizando uma amostra de, no mínimo, 1.599 pessoas..$^{15}$ Informações sobre o detalhamento metodológico da segunda onda do EpiFloripa Idoso podem ser verificadas no estudo de Schneider et al. ${ }^{16}$

0 desfecho do estudo - violência por parceiro íntimo - foi mensurado com o instrumento Conflict Tatics Scales Form R (CTS-1) em sua versão para 0 português, culturalmente adaptado ao contexto, para aferir a violência no casal. ${ }^{17} 0$ questionário investiga a violência psicológica, mediante seis itens classificados como 'xingamentos e ameaças' (xingou ou insultou; ficou emburrado; retirou-se do local; fez/disse coisas para irritar; ameaçou bater ou jogar coisas; destruiu/ jogou objetos), e a violência física, em oito itens identificados como 'força física ou explícita' (jogar objetos; empurrar/agarrar; dar tapas ou bofetadas; chutar, morder ou dar murro; bater ou tentar bater com objetos; espancar; estrangular/sufocar; ameaçar com faca ou arma). Em cada item do questionário, sobre violência física e violência psicológica, dicotomizado em 'sim' e 'não', questiona-se o entrevistado se cometeu 0 ato (violência perpetrada) ou se o parceiro cometeu contra ele (violência sofrida). Quando o mesmo indivíduo sofreu e perpetrou violência, definiu-se violência bidirecional. Foi considerada presença de violência por parceiro íntimo quando se obteve resposta positiva para ao menos um dos itens da escala. 0 período de recordatório que o CTS-1 utiliza se refere aos últimos 12 meses.
As variáveis exploratórias sociodemográficas foram: raça/cor da pele autorreferida (branca; preta; parda/amarela/indígena), status conjugal (casado/ com companheiro; solteiro; separado/divorciado; viúvo), com quem o idoso mora (sozinho; com cônjuge/ familiares), se havia dependentes da renda do idoso (não; sim) e se possuía trabalho remunerado atual (não; sim). A variável 'sexo' (feminino; masculino) foi utilizada para estratificação da análise.

As condições de saúde investigadas foram: autopercepção de saúde (muito boa/boa; regular; ruim/ muito ruim), consumo de bebida alcoólica (nunca; moderado; alto); hábito de fumar (nunca fumou; ex-fumante; fuma atualmente) e morbidades autorreferidas (nenhuma/uma; duas ou mais). 0 déficit cognitivo foi mensurado por meio do miniexame do estado mental de Folstein (MEEM), categorizado como provável déficit cognitivo (sim; não), com 0 uso da escolaridade para a definição dos pontos de corte.$^{18} \mathrm{~A}$ ocorrência de sintomas depressivos foi avaliada pela escala de depressão geriátrica (GDS-15) em sua versão reduzida, com 15 perguntas, em que indivíduos com seis ou mais pontos foram classificados como 'suspeito de depressão. ${ }^{19}$

A dependência das atividades de vida diária foi medida pela escala das atividades da vida diária (AVD), do Brazilian OARS Multidimensional Assessment Questionnaire (BOMFAQ), apta a mensurar a capacidade funcional com a proposição de 15 perguntas sobre atividades básicas da vida diária (ABVD) e atividades instrumentais de vida diária (AIVD) ${ }^{20}$ A classificação da dependência de AVD pautou-se em três categorias: ausência de incapacidade; leve, quando houve incapacidade/dificuldade para realizar entre uma e três AVDs; moderada/grave, quando houve incapacidade/dificuldade de realizar quatro ou mais AVDs.

Consideraram-se variáveis de ajuste: faixa etária (em anos: 60-69; 70-79; $\geq 80$ ), renda familiar per capita (em salários mínimos: até 1; entre 1 e 5 ; entre 5 e 10; maior que 10) e escolaridade (em anos de estudo: sem escolaridade formal; $1-4 ; 5-8 ; 9-11 ; \geq 12$ ).

As entrevistas foram realizadas entre novembro de 2013 e outubro de 2014, face a face, por entrevistadores treinados, e registradas em computadores portáteis (netbooks). As questões sobre violência, preferencialmente apresentadas à idosa ou idoso sozinho, se encontravam no último bloco do questionário, haja vista a natureza do tema e a expectativa de maior 
aproximação entre entrevistador e entrevistado. 0 controle de qualidade da coleta de dados foi feito por telefone, aplicando-se uma versão reduzida do questionário em $10 \%$ dos entrevistados, selecionados aleatoriamente.

No início da análise de dados, foi realizada estatística descritiva da amostra, comparando-se os idosos incluídos com os excluídos do estudo, para identificar as características de cada grupo. Também foi realizada análise descritiva das características sociodemográficas e condições de saúde dos participantes, com estratificação por sexo. A prevalência e respectivos intervalos de confiança de $95 \%\left(\mathrm{IC}_{95 \%}\right.$ ) da violência por parceiro íntimo foram descritos para os sexos masculino e feminino, de acordo com a natureza do ato: qualquer ação caracterizada como violência, física ou psicológica (violência física [isolada]; violência psicológica [isolada]; violência física e psicológica [concomitantes]); e a direcionalidade da violência (sofrida; perpetrada; bidirecional). Aplicou-se o teste qui-quadrado de Person e 0 teste exato de Fisher (para $n<20$ ), para comparação dos coeficientes de violência entre os sexos.

Para identificação dos fatores sociodemográficos e condições de saúde associados à violência por parceiro íntimo, considerou-se qualquer resposta positiva de violência, fosse física, psicológica ou bidirecional, como desfecho. Por regressão de Poisson, foram estimadas as razões de prevalências (RP) e $\mathrm{IC}_{95 \%}$, estratificados por sexo e direcionalidade da violência cometida por parceiro íntimo, em análise não ajustada e análise ajustada por idade, escolaridade e renda. Aplicaram-se dois modelos de regressão: violência sofrida e violência perpetrada, estratificados por sexo. Na análise ajustada, foi utilizado modelo hierárquico com dois níveis: o primeiro nível incluiu as variáveis sociodemográficas (raça/cor da pele, status conjugal, coabitação, existência dependente da renda do idoso, presença de trabalho remunerado atual); o segundo nível, as condições de saúde (autopercepção de saúde, frequência do consumo de álcool, hábito de fumar, comorbidades autorreferidas, déficit cognitivo, dependência de AVD). A inserção das variáveis no modelo ajustado seguiu os níveis estabelecidos: quando houve $\mathrm{p}$-valor $<0,20$ no modelo não ajustado, as variáveis foram inseridas no modelo ajustado, sendo consideradas estatisticamente significativas quando apresentaram p-valor $<0,05$ no modelo final. A análise dos dados foi realizada utilizando-se o software estatístico Stata 14.0 (Stata
Corporation, College Station, USA). Considerou-se o efeito do desenho amostral por conglomerados, sendo incorporados os pesos amostrais no módulo survey do programa Stata (comando svy).

0 projeto do estudo EpiFloripa Idoso foi aprovado pelo Comitê de Ética em Pesquisa com Seres Humanos da Universidade Federal de Santa Catarina (CEPSH/UFSC): Protocolo n ${ }^{0}$ 352/2008, emitido em 15 de dezembro de 2008; Certificado de Apresentação para Apreciação Ética (CAAE) n ${ }^{0}$ 16731313.0.0000.0121, de 10/02/2014. Todos os participantes assinaram Termos de Consentimento Livre e Esclarecido.

\section{Resultados}

Na primeira onda, foram entrevistados 1.702 idosos. Na segunda onda, houve $376(22,1 \%)$ perdas, totalizando 1.197 entrevistados em 2013/2014, com taxa de resposta de 70,3\%. Adicionalmente, para este estudo, foram excluídos 458 idosos por não possuírem parceiros nos últimos 12 meses, 57 entrevistas respondidas por informantes e 31 devido a recusas, totalizando 651 idosos entrevistados, com taxa de resposta de 54,4\% (Figura 1).

Ao se compararem os idosos incluídos $(\mathrm{n}=651)$ com os não incluídos ( $\mathrm{n}=546$ ), verificaram-se diferenças significativas, estatisticamente, em relação ao sexo, renda, escolaridade e condições de saúde. Dos não incluídos, $85,5 \%$ correspondiam ao sexo feminino, 53,6\% tinham até 4 anos de estudo e $65,1 \%$ referiram renda de até 5 salários mínimos. A proporção de idosos com déficit cognitivo (36,0\% vs 17,0\%), sintomas depressivos $(22,6 \%$ vs $17,6 \%)$ e algum grau de dependência em AVD $(74,5 \%$ vs 64,9$)$ foi superior entre os não incluídos, na comparação com os incluídos.

As características da amostra estão descritas na Tabela 1, estratificadas por sexo. No sexo masculino, 38,1\% (n=138 - $\left.\mathrm{IC}_{95 \%} 31,1 ; 45,0\right)$ apresentavam renda familiar entre 1 e 5 salários mínimos e 36,9\% $\left(\mathrm{n}=120-\mathrm{IC}_{95 \%} 29,8 ; 44,0\right)$ somavam 12 ou mais anos de estudo. No sexo feminino, $50,8 \%\left(\mathrm{n}=144-\mathrm{IC}_{95 \%}\right.$ $43,2 ; 58,3)$ referiam renda familiar entre 1 e 5 salários mínimos e $31,8 \%\left(\mathrm{n}=106-\mathrm{IC}_{95 \%} 24,6 ; 38,9\right)$ contavam $1 \mathrm{a} 4$ anos de estudo.

As principais características dos sexos masculino e feminino, respectivamente, foram: ser casado $(95,3 \%$ e $93,0 \%)$, raça/cor da pele branca $(84,0 \%$ e $88,1 \%)$, morar com cônjuge ou familiares $(95,4 \%$ e $96,0 \%)$, 


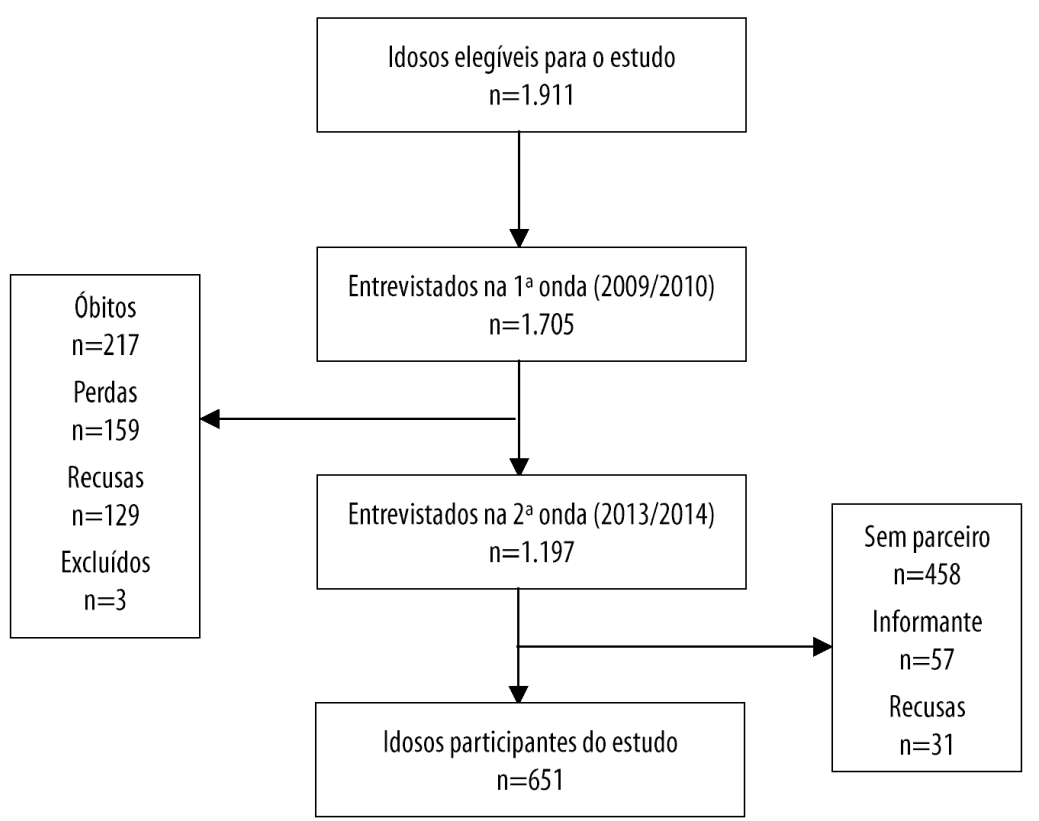

Figura 1 - Fluxograma do tamanho da amostra do estudo EpiFloripa, Florianópolis, Santa Catarina, 2013/2014

Tabela 1 - Características dos idosos ( $n=651$ ) segundo variáveis sociodemográficas e econômicas, estratificadas por sexo, Florianópolis, Santa Catarina, 2013/2014

\begin{tabular}{|c|c|c|c|c|}
\hline \multirow{2}{*}{ Variáveis } & \multicolumn{2}{|c|}{ Sexo masculino } & \multicolumn{2}{|c|}{ Sexo feminino } \\
\hline & n (\%) & IC $_{95 \%} \mathrm{a}^{\mathrm{a}}$ & $n(\%)$ & $\mathrm{IC}_{95 \%}{ }^{\mathrm{a}}$ \\
\hline Faixa etária (anos) & $n=340$ & & $n=311$ & \\
\hline $60-69$ & $135(37,5)$ & 30,$6 ; 44,4$ & $148(47,3)$ & 41,$0 ; 53,6$ \\
\hline $70-79$ & $146(45,1)$ & 37,$4 ; 52,8$ & $129(41,8)$ & 35,$5 ; 48,0$ \\
\hline$\geq 80$ & $59(17,4)$ & 12,$6 ; 22,2$ & $34(10,9)$ & 5,$8 ; 16,0$ \\
\hline Status conjugal & $n=340$ & & $n=311$ & \\
\hline Casado/com companheiro & $325(95,3)$ & 91,$8 ; 98,8$ & $288(93,0)$ & 89,$6 ; 96,3$ \\
\hline Solteiro & $4(0,9)$ & 0,$0 ; 1,9$ & $5(1,4)$ & 0,$0 ; 2,8$ \\
\hline Divorciado/separado & $7(1,8)$ & 0,$2 ; 3,3$ & $5(1,3)$ & 0,$1 ; 2,4$ \\
\hline Viúvo & $4(2,0)$ & 0,$0 ; 5,0$ & $13(4,3)$ & 1,$7 ; 6,9$ \\
\hline Raça/cor da pele & $n=340$ & & $n=311$ & \\
\hline Branca & $285(84,0)$ & 78,$4 ; 89,6$ & $273(88,1)$ & 82,$7 ; 93,6$ \\
\hline Preta & $32(9,5)$ & 5,$7 ; 13,4$ & $23(7,5)$ & 3,$0 ; 11,9$ \\
\hline Parda/amarela/indígena & $23(6,3)$ & 2,$3 ; 10,3$ & $14(4,3)$ & 1,$3 ; 7,3$ \\
\hline Coabitação & $n=339$ & & $n=311$ & \\
\hline Sozinho & $17(4,6)$ & 2,$1 ; 7,0$ & $13(4,0)$ & 1,$4 ; 6,6$ \\
\hline Cônjuge/familiares & $322(95,4)$ & 92,$9 ; 97,8$ & $297(96,0)$ & 93,$3 ; 98,6$ \\
\hline
\end{tabular}


Continuação

Tabela 1 - Características dos idosos ( $n=651$ ) segundo variáveis sociodemográficas e econômicas, estratificadas por sexo, Florianópolis, Santa Catarina, 2013/2014

\begin{tabular}{|c|c|c|c|c|}
\hline \multirow{2}{*}{ Variáveis } & \multicolumn{2}{|c|}{ Sexo masculino } & \multicolumn{2}{|c|}{ Sexo feminino } \\
\hline & n (\%) & $I_{95 \%}{ }^{a}$ & $n(\%)$ & IC $_{95 \%}{ }^{a}$ \\
\hline Escolaridade (anos de estudo) & $n=340$ & & $n=311$ & \\
\hline Sem escolaridade formal & $23(5,7)$ & 27,$2 ; 86,5$ & $13(3,2)$ & 13,$8 ; 49,7$ \\
\hline $1-4$ & $90(22,7)$ & 16,$8 ; 28,4$ & $106(31,8)$ & 24,$6 ; 38,9$ \\
\hline $5-8$ & $54(15,6)$ & 11,$4 ; 19,9$ & $59(18,9)$ & 13,$7 ; 23,9$ \\
\hline $9-11$ & $53(19,1)$ & 14,$6 ; 23,6$ & $58(20,1)$ & 13,$9 ; 26,4$ \\
\hline$\geq 12$ & $120(36,9)$ & 29,$8 ; 44,0$ & $75(26,0)$ & 19,$8 ; 32,2$ \\
\hline Renda familiar (em salários mínimos) & $n=329$ & & $\mathrm{n}=\mathbf{2 9 0}$ & \\
\hline Menor que 1 & $9(3,3)$ & 0,$0 ; 6,6$ & $15(4,2)$ & 1,$6 ; 6,7$ \\
\hline Entre 1 e 5 & $138(38,1)$ & 31,$1 ; 45,0$ & $144(50,8)$ & 43,$2 ; 58,3$ \\
\hline Entre 5 e 10 & $77(24,8)$ & 19,$4 ; 30,1$ & $76(26,1)$ & 20,$2 ; 32,0$ \\
\hline Maior que 10 & $105(33,8)$ & 26,$7 ; 40,9$ & $55(18,9)$ & 12,$7 ; 25,1$ \\
\hline Dependentes da renda & $\mathrm{n}=337$ & & $n=223$ & \\
\hline Não & $71(20,6)$ & 13,$4 ; 27,7$ & $129(54,8)$ & 46,$1 ; 63,3$ \\
\hline Sim & $266(79,4)$ & 72,$2 ; 86,5$ & $94(45,2)$ & 36,$6 ; 53,8$ \\
\hline Trabalho remunerado & $n=337$ & & $n=225$ & \\
\hline Não & $277(81,0)$ & 73,$8 ; 88,1$ & $201(90,0)$ & 85,$7 ; 94,2$ \\
\hline Sim & $60(19,0)$ & 11,$8 ; 26,2$ & $24(10,0)$ & 5,$8 ; 14,2$ \\
\hline Autopercepção de saúde & $\mathrm{n}=\mathbf{3 4 0}$ & & $n=311$ & \\
\hline Muito boa/boa & $226(68,2)$ & 62,$3 ; 74,2$ & $171(60,6)$ & 53,$4 ; 67,8$ \\
\hline Regular & $117(26,6)$ & 20,$8 ; 32,4$ & $117(33,4)$ & 27,$1 ; 39,6$ \\
\hline Ruim/muito ruim & $16(5,2)$ & 1,$6 ; 8,6$ & $23(6,0)$ & 30,$9 ; 89,4$ \\
\hline Sintomas depressivos & $\mathrm{n}=338$ & & $n=310$ & \\
\hline Normal & $289(85,8)$ & 80,$9 ; 90,6$ & $245(80,9)$ & 75,$2 ; 96,5$ \\
\hline Suspeita de depressão & $49(14,2)$ & 9,$3 ; 19,1$ & $65(19,1)$ & 13,$4 ; 24,8$ \\
\hline Déficit cognitivo & $n=340$ & & $\mathrm{n}=308$ & \\
\hline Negativo & $289(87,2)$ & 81,$3 ; 93,2$ & $246(80,0)$ & 74,$5 ; 85,4$ \\
\hline Positivo & $51(12,8)$ & 6,$7 ; 18,7$ & $62(20,0)$ & 14,$5 ; 25,4$ \\
\hline Dependência em AVD & $n=338$ & & $n=311$ & \\
\hline Ausência & $145(43,1)$ & 37,$5 ; 48,8$ & $83(26,5)$ & 20,$6 ; 32,5$ \\
\hline Leve & $134(39,7)$ & 33,$7 ; 45,6$ & $139(47,2)$ & 40,$7 ; 53,7$ \\
\hline Moderada/grave & $59(17,2)$ & 13,$1 ; 21,2$ & $89(26,3)$ & 21,$6 ; 30,9$ \\
\hline Morbidades & $n=340$ & & $n=311$ & \\
\hline Nenhuma/uma & $104(32,7)$ & 27,$2 ; 38,2$ & $57(20,7)$ & 14,$9 ; 26,4$ \\
\hline Duas ou mais & $236(67,3)$ & 61,$7 ; 72,8$ & $254(79,3)$ & 73,$5 ; 85,0$ \\
\hline
\end{tabular}


Continuação

Tabela 1 - Características dos idosos ( $n=651$ ) segundo variáveis sociodemográficas e econômicas, estratificadas por sexo, Florianópolis, Santa Catarina, 2013/2014

\begin{tabular}{|c|c|c|c|c|}
\hline \multirow{2}{*}{ Variáveis } & \multicolumn{2}{|c|}{ Sexo masculino } & \multicolumn{2}{|c|}{ Sexo feminino } \\
\hline & $\mathrm{n}(\%)$ & $\mathrm{IC}_{95 \%}{ }^{\mathrm{a}}$ & $\mathrm{n}(\%)$ & $I_{95 \%}{ }^{a}$ \\
\hline Consumo de bebida alcoólica & $n=340$ & & $n=311$ & \\
\hline Nunca & $128(35,1)$ & 27,$6 ; 42,6$ & $203(63,6)$ & 56,$3 ; 70,7$ \\
\hline Moderado & $95(29,3)$ & 23,$2 ; 35,4$ & $82(26,9)$ & 20,$0 ; 33,7$ \\
\hline Alto & $117(35,6)$ & 29,$0 ; 42,0$ & $26(9,5)$ & 4,$5 ; 14,5$ \\
\hline Hábito de fumar & $n=340$ & & $n=311$ & \\
\hline Nunca fumou & $116(32,7)$ & 26,$3 ; 39,1$ & $235(74,7)$ & 68,$8 ; 80,6$ \\
\hline Ex-fumante & $187(56,0)$ & 49,$3 ; 62,4$ & $62(21,1)$ & 49,$3 ; 62,4$ \\
\hline Fuma atualmente & $37(11,3)$ & 7,$0 ; 15,6$ & $14(4,2)$ & 1,$1 ; 7,2$ \\
\hline
\end{tabular}

a) $\mathrm{IC}_{95 \%}$ : Intervalo de confiança de 95\%; b) AVD: atividade da vida diária.

Tabela 2 - Prevalência de violência por parceiro íntimo em idosos ( $n=651)$ segundo tipologia e direcionalidade da violência, estratificada por sexo, Florianópolis, Santa Catarina, 2013/2014

\begin{tabular}{|c|c|c|c|c|c|}
\hline \multirow{2}{*}{ Tipo e direcionalidade da violência } & \multicolumn{2}{|c|}{ Sexo masculino $(n=340)$} & \multicolumn{2}{|c|}{ Sexo feminino $(n=311)$} & \multirow{2}{*}{ p-valor } \\
\hline & $\mathbf{n}$ & $\%\left(\mathrm{IC}_{95 \%}\right)^{\mathrm{a}}$ & $\mathbf{n}$ & $\%\left(\mathrm{IC}_{95 \%}\right)^{\mathrm{a}}$ & \\
\hline \multicolumn{6}{|c|}{ Qualquer ato de violência por parceiro íntimo } \\
\hline Sofrida & 162 & $48,3(42,0 ; 54,5)$ & 148 & $46,4(39,6 ; 53,2)$ & $0,47^{b}$ \\
\hline Perpetrada & 167 & $50,3(43,1 ; 57,3)$ & 144 & $45,1(38,5 ; 51,8)$ & $0,20^{\mathrm{b}}$ \\
\hline Bidirecional & 145 & $42,9(37,1 ; 48,7)$ & 125 & $38,4(31,8 ; 44,9)$ & $0,18^{b}$ \\
\hline \multicolumn{6}{|c|}{ Violência por parceiro íntimo - psicológica } \\
\hline Sofrida & 162 & $48,3(42,0 ; 54,5)$ & 144 & $44,8(38,0 ; 51,7)$ & $0,22^{b}$ \\
\hline Perpetrada & 166 & $49,8(42,8 ; 56,6)$ & 142 & $44,5(38,0 ; 50,9)$ & $0,17^{b}$ \\
\hline Bidirecional & 145 & $42,9(37,1 ; 48,7)$ & 123 & $37,7(31,2 ; 44,1)$ & $0,12^{b}$ \\
\hline \multicolumn{6}{|l|}{ Violência por parceiro íntimo - física } \\
\hline Sofrida & 10 & $2,2(0,7 ; 3,5)$ & 13 & $3,9(1,4 ; 6,5)$ & $0,26^{c}$ \\
\hline Perpetrada & 8 & $2,0(0,4 ; 3,5)$ & 12 & $3,6(0,9 ; 6,3)$ & $0,18^{c}$ \\
\hline Bidirecional & 4 & $0,8(0,0 ; 1,8)$ & 8 & $2,4(0,4 ; 4,5)$ & $0,15^{c}$ \\
\hline \multicolumn{6}{|c|}{ Violência por parceiro íntimo - física e psicológica } \\
\hline Sofrida & 10 & $2,2(0,7 ; 3,5)$ & 9 & $2,3(0,4 ; 4,3)$ & $0,57^{c}$ \\
\hline Perpetrada & 7 & $1,5(0,3 ; 2,7)$ & 10 & $3,0(0,6 ; 5,3)$ & 0,24 \\
\hline Bidirecional & 4 & $0,8(0,0 ; 1,8)$ & 6 & $1,8(0,0 ; 3,6)$ & $0,32^{c}$ \\
\hline
\end{tabular}

a) $I_{95 \%}$ : Intervalo de confiança de $95 \%$; b) Teste qui-quadrado de Pearson; c) Teste exato de Fisher. 
Tabela 3 - Razão de prevalências não ajustada de violência por parceiro íntimo, sofrida e perpetrada (n=651), segundo variáveis sociodemográficas, econômicas e condições de saúde, Florianópolis, Santa Catarina, 2013/2014

\begin{tabular}{|c|c|c|c|c|}
\hline \multirow{3}{*}{ Variáveis } & \multicolumn{2}{|c|}{ Violência sofrida } & \multicolumn{2}{|c|}{ Violência perpetrada } \\
\hline & Sexo masculino & Sexo feminino & Sexo masculino & Sexo feminino \\
\hline & $\mathrm{RPa}\left(\mathrm{IC}_{95 \%}\right)^{\mathrm{b}}$ & $\mathrm{RPa}^{\mathrm{a}}\left(\mathrm{IC}_{95 \%}\right)^{\mathrm{b}}$ & $\mathrm{RPa}\left(\mathrm{IC}_{95 \%}\right)^{\mathrm{b}}$ & $\mathrm{RPa}\left(\mathrm{IC}_{95 \%}{ }^{b}\right)$ \\
\hline \multicolumn{5}{|l|}{ Faixa etária (anos) } \\
\hline $60-69$ & 1,00 & 1,00 & 1,00 & 1,00 \\
\hline $70-79$ & $0,85(0,62 ; 1,16)$ & $0,94(0,69 ; 1,28)$ & $0,87(0,65 ; 1,14)$ & $0,68(0,48 ; 0,97)$ \\
\hline$\geq 80$ & $0,69(0,45 ; 1,07)$ & $0,93(0,57 ; 1,52)$ & $0,59(0,37 ; 0,93)$ & $0,53(0,29 ; 0,95)$ \\
\hline \multicolumn{5}{|l|}{ Raça/cor da pele } \\
\hline Branca & 1,00 & 1,00 & 1,00 & 1,00 \\
\hline Preta & $1,14(0,79 ; 1,64)$ & $0,65(0,35 ; 1,21)$ & $0,92(0,51 ; 1,41)$ & $0,62(0,33 ; 1,16)$ \\
\hline Parda/amarela/indígena & $1,16(0,64 ; 2,11)$ & $1,34(0,89 ; 2,02)^{c}$ & $1,43(1,06 ; 1,93)^{c}$ & $1,38(0,90 ; 2,11)^{c}$ \\
\hline \multicolumn{5}{|l|}{ Status conjugal } \\
\hline Casado & 1,00 & 1,00 & 1,00 & 1,00 \\
\hline Solteiro & $0,54(0,11 ; 2,52)$ & $0,89(0,24 ; 3,34)$ & $1,24(0,53 ; 2,92)$ & $0,92(0,24 ; 3,43)$ \\
\hline Divorciado/separado & $0,42(0,10 ; 1,72)$ & $0,98(0,35 ; 2,73)$ & $0,74(0,34 ; 1,64)$ & $1,87(1,31 ; 2,66)$ \\
\hline Viúvo & $0,13(0,01 ; 1,53)^{\mathrm{a}}$ & $0,79(0,33 ; 1,91)$ & $1,66(1,12 ; 2,47) c$ & $0,45(0,16 ; 1,27)^{c}$ \\
\hline \multicolumn{5}{|l|}{ Coabitação } \\
\hline Sozinho & 1,00 & 1,00 & 1,00 & 1,00 \\
\hline Acompanhado & $1,52(0,68 ; 3,39)$ & $1,11(0,48 ; 2,57)$ & $1,11(0,61 ; 20,1)$ & $1,93(0,72 ; 5,19)^{c}$ \\
\hline \multicolumn{5}{|c|}{ Escolaridade (anos de estudo) } \\
\hline Sem escolaridade formal & 1,00 & 1,00 & 1,00 & 1,00 \\
\hline $1-4$ & $0,78(0,47 ; 1,30)$ & $0,94(0,50 ; 1,76)$ & $0,84(0,50 ; 1,41)$ & $1,03(0,53 ; 1,99)$ \\
\hline $5-8$ & $0,90(0,52 ; 1,54)$ & $0,68(0,32 ; 1,42)$ & $1,10(0,65 ; 1,86)$ & $0,79(0,38 ; 1,66)$ \\
\hline 9-11 & $0,73(0,39 ; 1,37)$ & $1,00(0,52 ; 1,92)$ & $0,96(0,53 ; 1,73)$ & $1,09(0,51 ; 2,32)$ \\
\hline$\geq 12$ & $0,86(0,52 ; 1,41)$ & $0,87(0,45 ; 1,67)$ & $0,89(0,55 ; 1,46)$ & $0,90(0,46 ; 1,76)$ \\
\hline \multicolumn{5}{|c|}{ Renda familiar (em salários mínimos) } \\
\hline Menor que 1 & 1,00 & 1,00 & 1,00 & 1,00 \\
\hline Entre 1 e 5 & $2,50(0,47 ; 13,0)$ & $1,12(0,64 ; 1,97)$ & $3,94(0,84 ; 18,44)$ & $1,06(0,60 ; 1,88)$ \\
\hline Entre 5 e 10 & $2,15(0,39 ; 11,62)$ & $0,87(0,51 ; 1,48)$ & $3,98(0,86 ; 18,38)$ & $0,90(0,52 ; 1,58)$ \\
\hline Maior que 10 & $2,57(0,50 ; 12,97)$ & $0,86(0,44 ; 1,68)$ & $3,99(0,84 ; 19,03)$ & $0,88(0,41 ; 1,88)$ \\
\hline \multicolumn{5}{|l|}{ Dependentes da renda } \\
\hline Não & 1,00 & 1,00 & 1,00 & 1,00 \\
\hline Sim & $1,07(0,78 ; 1,47)$ & $1,02(0,71 ; 1,45)$ & $0,89(0,62 ; 1,28)$ & $1,13(0,82 ; 1,55)$ \\
\hline \multicolumn{5}{|l|}{ Trabalho remunerado } \\
\hline Não & 1,00 & 1,00 & 1,00 & 1,00 \\
\hline Sim & $0,79(0,54 ; 1,16)$ & $1,27(0,78 ; 2,05)$ & $1,19(0,81 ; 1,75)$ & $1,22(0,76 ; 1,95)$ \\
\hline \multicolumn{5}{|l|}{ Autopercepção de saúde } \\
\hline Muito boa/boa & 1,00 & 1,00 & 1,00 & 1,00 \\
\hline Regular & $1,14(0,88 ; 1,47)$ & $1,44(1,02 ; 2,03)$ & $1,05(0,80 ; 1,38)$ & $1,67(1,24 ; 2,25)$ \\
\hline Ruim/muito ruim & $0,92(0,39 ; 2,18)$ & $0,98(0,52 ; 1,87)^{c}$ & $1,73(1,25 ; 2,40)^{c}$ & $1,04(0,58 ; 1,89)^{c}$ \\
\hline
\end{tabular}


Continuação

Tabela 3 - Razão de prevalências não ajustada de violência por parceiro íntimo, sofrida e perpetrada ( $n=651$ ), segundo variáveis sociodemográficas, econômicas e condições de saúde, Florianópolis, Santa Catarina, 2013/2014

\begin{tabular}{|c|c|c|c|c|}
\hline \multirow{3}{*}{ Variáveis } & \multicolumn{2}{|c|}{ Violência sofrida } & \multicolumn{2}{|c|}{ Violência perpetrada } \\
\hline & Sexo masculino & Sexo feminino & Sexo masculino & Sexo feminino \\
\hline & $\mathrm{RPa}\left(\mathrm{IC}_{95 \%}\right)^{\mathrm{b}}$ & $\mathrm{RPa}^{\mathrm{a}}\left(\mathrm{IC}_{95 \%}\right)^{\mathrm{b}}$ & $R P a\left(I C_{95 \%}\right)^{b}$ & $\mathrm{RPa}\left(\mathrm{IC}_{95 \%}{ }^{\mathrm{b}}\right)$ \\
\hline \multicolumn{5}{|l|}{ Sintomas depressivos } \\
\hline Normal & 1,00 & 1,00 & 1,00 & 1,00 \\
\hline Suspeita de depressão & $0,96(0,64 ; 1,46)$ & $1,13(0,77 ; 1,65)$ & $1,14(0,84 ; 1,55)$ & $1,32(0,88 ; 1,97)^{c}$ \\
\hline \multicolumn{5}{|l|}{ Déficit cognitivo } \\
\hline Negativo & 1,00 & 1,00 & 1,00 & 1,00 \\
\hline Positivo & $0,95(0,71 ; 1,27)$ & $0,89(0,59 ; 1,34)$ & $0,74(0,48 ; 1,13)^{c}$ & $1,03(0,68 ; 1,56)$ \\
\hline \multicolumn{5}{|l|}{ Dependência em AVD ${ }^{b}$} \\
\hline Nenhuma & 1,00 & 1,00 & 1,00 & 1,00 \\
\hline 1 a 3 & $0,99(0,72 ; 1,35)$ & $0,98(0,68 ; 1,41)$ & $0,86(0,64 ; 1,15)$ & $0,94(0,67 ; 1,32)$ \\
\hline 4 ou mais & $1,00(0,64 ; 1,57)$ & $1,11(0,75 ; 1,66)$ & $1,15(0,84 ; 1,56)$ & $1,05(0,76 ; 1,44)$ \\
\hline \multicolumn{5}{|l|}{ Morbidades } \\
\hline Nenhuma/uma & 1,00 & 1,00 & 1,00 & 1,00 \\
\hline Duas ou mais & $1,01(0,81 ; 1,25)$ & $1,14(0,81 ; 1,61)$ & $1,19(0,93 ; 1,52)^{c}$ & $0,97(0,67 ; 1,42)$ \\
\hline \multicolumn{5}{|c|}{ Consumo de bebida alcoólica } \\
\hline Nunca & 1,00 & 1,00 & 1,00 & 1,00 \\
\hline Moderado & $1,33(0,98 ; 1,81)$ & $1,20(0,93 ; 1,56)$ & $1,00(0,72 ; 1,40)$ & $1,19(0,90 ; 1,59)$ \\
\hline Alto & $1,21(0,87 ; 1,70)^{c}$ & $1,23(0,81 ; 189)$ & $1,12(0,80 ; 1,58)$ & $1,27(0,81 ; 1,99)$ \\
\hline \multicolumn{5}{|l|}{ Hábito de fumar } \\
\hline Nunca fumou & 1,00 & 1,00 & 1,00 & 1,00 \\
\hline Ex-fumante & $0,93(0,71 ; 1,22)$ & $1,01(0,76 ; 1,34)$ & $1,05(0,76 ; 1,44)$ & $0,96(0,70 ; 1,33)$ \\
\hline Fuma atualmente & $0,88(0,52 ; 1,47)$ & $1,42(0,97 ; 2,09)^{c}$ & $1,08(0,73 ; 1,61)$ & $1,05(0,58 ; 1,87)$ \\
\hline
\end{tabular}

a) RP: Razão de prevalências; b) IC $\mathrm{C}_{95 \%}$ : Intervalo de confiança de 95\%; c) P-valor <0,20; d) AVD: Atividade da vida diária.

não ter trabalho atual remunerado (81,0\% e 90,0\%), ter autopercepção de saúde muito boa/boa (68,2\% e $60,6 \%)$, possuir duas ou mais morbidades $(67,3 \%$ e $79,3 \%)$, não ter sintomas depressivos (85,8\% e 80,9\%) e tampouco déficit cognitivo $(87,2 \%$ e $80,0 \%)$. Não houve dependência em AVD para 43,1\% da amostra do sexo masculino $\left(\mathrm{n}=145-\mathrm{IC}_{95 \%} 37,5 ; 48,8\right)$, enquanto, no sexo feminino, $47,2 \%\left(\mathrm{n}=139-\mathrm{IC}_{95 \%} 40,7 ; 53,7\right)$ revelaram essa dependência em grau leve. Quanto a bebida alcoólica e tabagismo, 63,3\% ( $\mathrm{n}=203-\mathrm{IC}_{95 \%}$ $56,3 ; 70,7)$ das idosas relataram nunca ter bebido e $74,7 \%$ ( $\left.\mathrm{n}=235-\mathrm{IC}_{95 \%} 68,8 ; 80,6\right)$ nunca ter fumado, ao passo que, nos idosos homens, 35,6\% ( $\mathrm{n}=117$ $\left.\mathrm{IC}_{95 \%} 29,0 ; 42,0\right)$ referiram consumo alto de bebida alcóolica e 56,0\% ( $\left.\mathrm{n}=187-\mathrm{IC}_{95 \%} 49,3 ; 62,4\right)$ eram ex-fumantes (Tabela 1).
A violência psicológica predominou entre os atos de violência por parceiro íntimo, fosse ela sofrida (48,3\% e $44,8 \%)$ ou perpetrada (49,8\% e $44,5 \%)$, quando comparada à violência física sofrida $(2,2 \%$ e $3,9 \%)$ ou perpetrada (2,0\% e 3,6\%) (estas, em menores proporções), nos idosos do sexo masculino e do sexo feminino respectivamente. A maioria das pessoas idosas em situação de violência física também estiveram expostas à violência psicológica por parceiro íntimo, evidenciando-se a sobreposição das violências (Tabela 2). Destaca-se que não houve diferença entre as prevalências dos diferentes tipos de violência por parceiro íntimo sofrida, perpetrada e bidirecional, quando estratificada por sexo.

$\mathrm{Na}$ análise não ajustada, no sexo masculino, a autopercepção de saúde ruim/muito ruim associou-se à 
Tabela 4 - Razão de prevalências ajustada de violência por parceiro íntimo sofrida e perpetrada por idosos ( $n=651$ ), segundo variáveis sociodemográficas, econômicas e condições de saúde, Florianópolis, Santa Catarina, 2013/2014

\begin{tabular}{|c|c|c|c|c|}
\hline \multirow{3}{*}{ Variáveis } & \multicolumn{2}{|c|}{ Violência sofrida } & \multicolumn{2}{|c|}{ Violência perpetrada } \\
\hline & Sexo masculino & Sexo feminino & Sexo masculino & Sexo feminino \\
\hline & $\operatorname{RPa}\left(\mathbf{I C}_{95 \%}\right)^{\mathrm{b}}$ & $\operatorname{RPa}\left(\mathrm{IC}_{95 \%}\right)^{\mathrm{b}}$ & $\mathrm{RPa}\left(\mathrm{IC}_{95 \%}\right)^{\mathrm{b}}$ & $\operatorname{RPa}\left(I_{95 \%}\right)^{b}$ \\
\hline \multicolumn{5}{|l|}{ Faixa etária (anos) } \\
\hline $60-69$ & 1,00 & 1,00 & 1,00 & 1,00 \\
\hline 70-79 & $0,85(0,62 ; 1,16)$ & $0,94(0,69 ; 1,28)$ & $0,87(0,65 ; 1,14)$ & $0,68(0,48 ; 0,97)$ \\
\hline$\geq 80$ & $0,69(0,45 ; 1,07)$ & $0,93(0,57 ; 1,52)$ & $0,59(0,37 ; 0,93)$ & $0,53(0,29 ; 0,95)$ \\
\hline \multicolumn{5}{|l|}{ Raça/cor da pele } \\
\hline Branca & 1,00 & 1,00 & 1,00 & 1,00 \\
\hline Preta & $1,14(0,79 ; 1,64)$ & $0,65(0,35 ; 1,21)$ & $0,92(0,51 ; 1,41)$ & $0,62(0,33 ; 1,16)$ \\
\hline Parda/amarela/indígena & $1,16(0,64 ; 2,11)$ & $1,34(0,89 ; 2,02)^{c}$ & $1,43(1,06 ; 1,93)^{c}$ & $1,38(0,90 ; 2,11)^{c}$ \\
\hline \multicolumn{5}{|l|}{ Status conjugal } \\
\hline Casado & 1,00 & 1,00 & 1,00 & 1,00 \\
\hline Solteiro & $0,54(0,11 ; 2,52)$ & $0,89(0,24 ; 3,34)$ & $1,24(0,53 ; 2,92)$ & $0,92(0,24 ; 3,43)$ \\
\hline Divorciado/separado & $0,42(0,10 ; 1,72)$ & $0,98(0,35 ; 2,73)$ & $0,74(0,34 ; 1,64)$ & $1,87(1,31 ; 2,66)$ \\
\hline Viúvo & $0,13(0,01 ; 1,53)^{\mathrm{a}}$ & $0,79(0,33 ; 1,91)$ & $1,66(1,12 ; 2,47) c$ & $0,45(0,16 ; 1,27)^{c}$ \\
\hline \multicolumn{5}{|l|}{ Coabitação } \\
\hline Sozinho & 1,00 & 1,00 & 1,00 & 1,00 \\
\hline Acompanhado & $1,52(0,68 ; 3,39)$ & $1,11(0,48 ; 2,57)$ & $1,11(0,61 ; 20,1)$ & $1,93(0,72 ; 5,19)^{c}$ \\
\hline \multicolumn{5}{|c|}{ Escolaridade (anos de estudo) } \\
\hline Sem escolaridade formal & 1,00 & 1,00 & 1,00 & 1,00 \\
\hline $1-4$ & $0,78(0,47 ; 1,30)$ & $0,94(0,50 ; 1,76)$ & $0,84(0,50 ; 1,41)$ & $1,03(0,53 ; 1,99)$ \\
\hline $5-8$ & $0,90(0,52 ; 1,54)$ & $0,68(0,32 ; 1,42)$ & $1,10(0,65 ; 1,86)$ & $0,79(0,38 ; 1,66)$ \\
\hline 9-11 & $0,73(0,39 ; 1,37)$ & $1,00(0,52 ; 1,92)$ & $0,96(0,53 ; 1,73)$ & $1,09(0,51 ; 2,32)$ \\
\hline$>12$ & $0,86(0,52 ; 1,41)$ & $0,87(0,45 ; 1,67)$ & $0,89(0,55 ; 1,46)$ & $0,90(0,46 ; 1,76)$ \\
\hline \multicolumn{5}{|c|}{ Renda familiar (em salários mínimos) } \\
\hline Menor que 1 & 1,00 & 1,00 & 1,00 & 1,00 \\
\hline Entre 1 e 5 & $2,50(0,47 ; 13,0)$ & $1,12(0,64 ; 1,97)$ & $3,94(0,84 ; 18,44)$ & $1,06(0,60 ; 1,88)$ \\
\hline Entre 5 e 10 & $2,15(0,39 ; 11,62)$ & $0,87(0,51 ; 1,48)$ & $3,98(0,86 ; 18,38)$ & $0,90(0,52 ; 1,58)$ \\
\hline Maior que 10 & $2,57(0,50 ; 12,97)$ & $0,86(0,44 ; 1,68)$ & $3,99(0,84 ; 19,03)$ & $0,88(0,41 ; 1,88)$ \\
\hline \multicolumn{5}{|l|}{ Dependentes da renda } \\
\hline Não & 1,00 & 1,00 & 1,00 & 1,00 \\
\hline Sim & $1,07(0,78 ; 1,47)$ & $1,02(0,71 ; 1,45)$ & $0,89(0,62 ; 1,28)$ & $1,13(0,82 ; 1,55)$ \\
\hline \multicolumn{5}{|l|}{ Trabalho remunerado } \\
\hline Não & 1,00 & 1,00 & 1,00 & 1,00 \\
\hline Sim & $0,79(0,54 ; 1,16)$ & $1,27(0,78 ; 2,05)$ & $1,19(0,81 ; 1,75)$ & $1,22(0,76 ; 1,95)$ \\
\hline \multicolumn{5}{|c|}{ Autopercepção de saúde } \\
\hline Muito boa/boa & 1,00 & 1,00 & 1,00 & 1,00 \\
\hline Regular & $1,14(0,88 ; 1,47)$ & $1,44(1,02 ; 2,03)$ & $1,05(0,80 ; 1,38)$ & $1,67(1,24 ; 2,25)$ \\
\hline Ruim/muito ruim & $0,92(0,39 ; 2,18)$ & $0,98(0,52 ; 1,87)^{c}$ & $1,73(1,25 ; 2,40)^{c}$ & $1,04(0,58 ; 1,89)^{c}$ \\
\hline
\end{tabular}


Continuação

Tabela 4 - Razão de prevalências ajustada de violência por parceiro íntimo sofrida e perpetrada por idosos ( $n=651$ ), segundo variáveis sociodemográficas, econômicas e condições de saúde, Florianópolis, Santa Catarina, 2013/2014

\begin{tabular}{|c|c|c|c|c|}
\hline \multirow{3}{*}{ Variáveis } & \multicolumn{2}{|c|}{ Violência sofrida } & \multicolumn{2}{|c|}{ Violência perpetrada } \\
\hline & Sexo masculino & Sexo feminino & Sexo masculino & Sexo feminino \\
\hline & $\operatorname{RP}^{\mathrm{a}}\left(\mathbf{I C}_{95 \%}\right)^{\mathrm{b}}$ & $\operatorname{RP}^{\mathrm{a}}\left(\mathrm{IC}_{95 \%}\right)^{\mathrm{b}}$ & $\operatorname{RP}^{\mathrm{a}}\left(\mathrm{IC}_{95 \%}\right)^{\mathrm{b}}$ & $\operatorname{RP}^{\mathrm{a}}\left(\mathrm{IC}_{95 \%}\right)^{\mathrm{b}}$ \\
\hline \multicolumn{5}{|l|}{ Sintomas depressivos } \\
\hline Normal & 1,00 & 1,00 & 1,00 & 1,00 \\
\hline Suspeita de depressão & $0,96(0,64 ; 1,46)$ & $1,13(0,77 ; 1,65)$ & $1,14(0,84 ; 1,55)$ & $1,32(0,88 ; 1,97)^{c}$ \\
\hline \multicolumn{5}{|l|}{ Déficit cognitivo } \\
\hline Negativo & 1,00 & 1,00 & 1,00 & 1,00 \\
\hline Positivo & $0,95(0,71 ; 1,27)$ & $0,89(0,59 ; 1,34)$ & $0,74(0,48 ; 1,13)^{c}$ & $1,03(0,68 ; 1,56)$ \\
\hline \multicolumn{5}{|l|}{ Dependência em AVD ${ }^{b}$} \\
\hline Nenhuma & 1,00 & 1,00 & 1,00 & 1,00 \\
\hline 1 a 3 & $0,99(0,72 ; 1,35)$ & $0,98(0,68 ; 1,41)$ & $0,86(0,64 ; 1,15)$ & $0,94(0,67 ; 1,32)$ \\
\hline 4 ou mais & $1,00(0,64 ; 1,57)$ & $1,11(0,75 ; 1,66)$ & $1,15(0,84 ; 1,56)$ & $1,05(0,76 ; 1,44)$ \\
\hline \multicolumn{5}{|l|}{ Morbidades } \\
\hline Nenhuma/uma & 1,00 & 1,00 & 1,00 & 1,00 \\
\hline Duas ou mais & $1,01(0,81 ; 1,25)$ & $1,14(0,81 ; 1,61)$ & $1,19(0,93 ; 1,52)^{c}$ & $0,97(0,67 ; 1,42)$ \\
\hline \multicolumn{5}{|c|}{ Consumo de bebida alcoólica } \\
\hline Nunca & 1,00 & 1,00 & 1,00 & 1,00 \\
\hline Moderado & $1,33(0,98 ; 1,81)$ & $1,20(0,93 ; 1,56)$ & $1,00(0,72 ; 1,40)$ & $1,19(0,90 ; 1,59)$ \\
\hline Alto & $1,21(0,87 ; 1,70)^{c}$ & $1,23(0,81 ; 189)$ & $1,12(0,80 ; 1,58)$ & $1,27(0,81 ; 1,99)$ \\
\hline \multicolumn{5}{|l|}{ Hábito de fumar } \\
\hline Nunca fumou & 1,00 & 1,00 & 1,00 & 1,00 \\
\hline Ex-fumante & $0,93(0,71 ; 1,22)$ & $1,01(0,76 ; 1,34)$ & $1,05(0,76 ; 1,44)$ & $0,96(0,70 ; 1,33)$ \\
\hline Fuma atualmente & $0,88(0,52 ; 1,47)$ & $1,42(0,97 ; 2,09)^{c}$ & $1,08(0,73 ; 1,61)$ & $1,05(0,58 ; 1,87)$ \\
\hline
\end{tabular}

a) RP: Razão de prevalências do modelo final, ajustado por idade, escolaridade e renda; b) $\mathrm{I}_{95 \%}$ : Intervalo de confiança de $95 \%$; c) P-valor $<0,05$.

violência perpetrada $\left(\mathrm{RP}=1,73-\mathrm{IC}_{95 \%} 1,25 ; 2,40\right)$. No sexo feminino, o status conjugal de divorciada/separada $\left(\mathrm{RP}=1,87-\mathrm{IC}_{95 \%} 1,31 ; 2,66\right)$ e a autopercepção de saúde regular $\left(\mathrm{RP}=1,67-\mathrm{IC}_{95 \%} 1,24 ; 2,25\right)$ associaram-se à prevalência da violência perpetrada. Não houve associação entre as variáveis investigadas e a violência sofrida, em idosos de ambos os sexos (Tabela 3).

$\mathrm{Na}$ análise ajustada, as associações encontradas no modelo não ajustado mantiveram-se semelhantes. Houve associação entre a prevalência de violência perpetrada por parceiro íntimo naqueles do sexo masculino com autopercepção de saúde ruim/muito ruim $\left(\mathrm{RP}=1,74-\mathrm{IC}_{95 \%}, 1,18 ; 2,58\right)$. Indivíduos do sexo feminino com status conjugal de separada/divorciada $\left(\mathrm{RP}=1,86-\mathrm{IC}_{95 \%} 1,15 ; 3,02\right)$ e com autopercepção de saúde regular $\left(\mathrm{RP}=1,53-\mathrm{IC}_{95 \%}\right.$ 1,12;2,08) tiveram associação positiva com a violência perpetrada por parceiro íntimo (Tabela 4).

\section{Discussão}

Elevadas prevalências de violência por parceiro íntimo sofrida, perpetrada e bidirecional foram encontradas entre mulheres e homens idosos, sem diferença entre os sexos. Constatou-se uma simetria de gênero na violência por parceiro íntimo, em todas as direcionalidades da violência analisadas. Houve maior prevalência da violência perpetrada em mulheres idosas divorciadas/separadas, com autopercepção de saúde regular, e em homens idosos com autopercepção de saúde ruim/muito ruim. 
Algumas limitações foram identificadas neste estudo. Seu desenho transversal impede a identificação das relações de causalidade entre as variáveis exploradas. A não investigação da ocorrência do abuso econômico e do comportamento controlador entre parceiros pode implicar subestimação da prevalência em idosos. As perdas elevadas, pela ausência de parceiros entre muitos entrevistados, ocasionaram redução da taxa de resposta na amostragem do estudo, bem como os vieses identificados nas perdas.

Ressalta-se que diferenças entre idosos incluídos e não incluídos na amostra do estudo podem limitar a identificação dos fatores associados à violência por parceiro íntimo, considerando-se que os idosos não participantes do estudo possuíam maiores vulnerabilidades socioeconômicas (escolaridade e renda inferior) e de saúde, tais como maiores prevalências de déficit cognitivo, sintomas depressivos e dependência em AVD. Quanto ao objeto de estudo, violência por parceiro íntimo, reconhece-se que públicos mais vulneráveis podem estar mais expostos à violência e, nesses casos, o fenômeno seria de mais difícil detecção. Destaca-se a escassez de estudos nacionais sobre a violência por parceiro íntimo em idosos, o que prejudicou sua comparabilidade com investigações sobre 0 tema em cenários culturais semelhantes no Brasil, centralizando a discussão dos resultados da pesquisa em sua comparação com os achados de estudos internacionais.

Quanto à predominância de violência psicológica sobre violência física, um estudo realizado nos Estados Unidos, em 2010, investigou as prevalências de violência contra idosos acima de 70 anos. ${ }^{21}$ Seus resultados corroboram os do presente trabalho, ao identificar 12,1\% para psicológica e 1,7\% para violência física, em idosos de ambos os sexos. Na maioria dos casos de violência física sofrida, também houve violência psicológica, indicando sobreposição das agressões. Mais um estudo norte-americano, focado na prevalência e características da violência por parceiro íntimo, sofrida por mulheres com mais de 65 anos de idade, corroborou a sobreposição de violências observada em Florianópolis: de acordo com esse estudo, 68,5\% das mulheres que relataram o comportamento controlador do parceiro também foram acometidas por outros tipos de abusos. ${ }^{11}$

A violência física e psicológica perpetrada por ambos os sexos, em proporções semelhantes, é reafirmada por estudo da Bélgica, com amostra nacional de base populacional, realizado em 2009. ${ }^{22}$
No entanto, outros estudos que incluíram idosos e analisaram a violência por parceiro íntimo, estratificada por sexo, encontraram maior prevalência de violência física contra mulheres e violência psicológica contra homens. ${ }^{23}$ As proporções semelhantes de violência por parceiro íntimo identificadas são confirmadas por mais um estudo ${ }^{24}$ com população adulta de Florianópolis (EpiFloripa Adulto 2009/2010), segundo o qual mulheres cometeram tantos atos de violência física moderada quanto homens. Dessa forma, sugere-se que a violência por parceiro íntimo não é fato novo na terceira idade, mas, isso sim, ocorre e se perpetua ao longo da vida.

Em relação ao achado de 'simetria de gênero', depara-se com um cenário sob o qual a violência está enraizada, seja nas estruturas sociais, seja no contexto familiar. Entretanto, o comportamento e as atitudes violentas utilizados para resolução dos conflitos, reconhecidamente presentes na violência entre casais, ${ }^{25}$ distanciam-se do entendimento comum do binômio 'vítima-agressor.' ${ }^{26}$ Tal simetria não representa, necessariamente, situações iguais em relação à frequência, motivações e consequências das agressões. ${ }^{27}$ Estudo nacional, conduzido na mesma capital Florianópolis, ao investigar a associação entre a qualidade de vida e a exposição à violência por parceiro íntimo entre idosos, mostrou impactos desiguais sobre os sexos: apenas as mulheres tiveram sua qualidade de vida comprometida quando foram vítimas e/ou perpetradoras dessa violência. ${ }^{28}$ Entende-se que diferenças entre as experiências de mulheres e homens em situação de violência certamente existem, e, para que se alcance uma compreensão detalhada do fenômeno, é preciso investigar esse desfecho em ambos os sexos.

As elevadas prevalências de violência por parceiro íntimo, encontradas neste trabalho, contradizem achados que, frequentemente, identificam familiares e cuidadores como principais agressores de pessoas idosas. Estudo sobre risco de violência contra idosos conduzido em Florianópolis, parte da segunda onda do projeto EpiFloripa Idoso (2013/2014), ${ }^{13}$ identificou o cônjuge como agressor, inserido na categoria de 'familiar', porém sem abordar, especificamente, a violência por parceiro íntimo. A violência no relacionamento íntimo entre idosos requer um olhar atento, haja vista a terceira idade trazer questões de enfrentamento decorrentes das condições de saúde próprias do envelhecimento, inclusive o aumento da proporção de suicídios. ${ }^{29}$ 
Em relação aos fatores associados, a predominância das análises unilaterais da violência, em que são investigadas apenas vítimas, indica que as correlações desses fatores com a perpetração da violência por parceiro íntimo ainda sejam subanalisadas, e pouco exploradas na literatura.

A propósito, uma revisão sistemática sobre estudos de base populacional, realizada em 2017, identificou associação entre violência por parceiro íntimo com, (i) 0 uso de álcool e (ii) o comprometimento funcional. ${ }^{10}$ Tais associações não foram encontradas no presente trabalho. Sobre o uso de álcool, embora pesquisas com idosos associem esse comportamento à violência por parceiro íntimo, ${ }^{10}$ estudo de coorte (EpiFloripa Idoso, 2009/2010 e 2013/2014) conduzido em Florianópolis apontou que a maioria dos entrevistados $(64,2 \%)$, quando questionados sobre a frequência do consumo bebidas alcoólicas, referiram 'nunca' fazer uso delas; e também identificou alta prevalência de morbidades $(56,7 \%)$ e de risco cardiovascular substancialmente aumentado $(52,4 \%) .{ }^{30}$ Pressupõe-se que, diante desse contexto, o uso frequente de medicamentos e as restrições médicas levem os idosos a ingerir álcool em menores proporções.

0 fato de 0 comprometimento funcional não se apresentar como fator associado, no presente estudo, pode estar relacionado à redução da violência física seguida do aumento da violência psicológica, a depender das limitações físicas presentes. Porém, chama a atenção a perpetuação do comportamento violento no envelhecimento, embora se altere, na forma que se manifesta ao longo da vida.

Sobre os fatores associados, faz-se necessária a inclusão de variáveis proximais do desfecho nos modelos de análise, por exemplo: exposição pregressa à violência, características pessoais referentes à personalidade e comportamento, ou satisfação no relacionamento íntimo. Ademais, sugere-se que fatores associados à violência por parceiro íntimo, comumente utilizados, sejam verificados em estudos conduzidos com a população adulta, e não sejam os mais relevantes quando se trata de idosos. Assim, recomenda-se estabelecer modelos conceituais exclusivos para a idade mais avançada.

0 estudo apresentado é relevante, ao analisar a violência por parceiro íntimo em um grupo etário pouco explorado na literatura. Tanto a prevalência como a análise dos fatores associados foram conduzidas de maneira estratificada, por sexo e direcionalidade da violência. Tais distinções permitem aprofundar o conhecimento da temática, a ocorrência e a distribuição da violência entre os idosos. 0 uso de período recordatório do último ano contribui para prevenir o viés de memória e, consequentemente, reduzir o sub-registro da prevalência, além de evitar sobreposições da violência ocorrida em outros períodos da vida.

A investigação de violência por parceiro íntimo a partir de amostra representativa de base populacional, por meio de um instrumento amplamente validado e de alta confiabilidade, o Conflict Tactics Scales Form $R$ (CTS-1), favorece a obtenção de resultados fidedignos que apontam para simetria de gênero, relativamente ao envolvimento nas situações de violência no relacionamento de parceiros íntimos. Os resultados encontrados também mostram a necessidade de intensificar políticas públicas que possibilitem 0 enfrentamento da violência por parceiro íntimo na terceira idade.

\section{Contribuição das autoras}

Warmling D, Lindner SR e Coelho EBS planejaram o delineamento da pesquisa, analisaram os dados, revisaram o texto e elaboraram a versão preliminar do manuscrito. Conceição TB contribuiu para a análise e interpretação dos dados, e participou na redação e revisão do conteúdo. Todas as autoras aprovaram a versão final do artigo e são responsáveis por todo o conteúdo do trabalho, inclusive por garantir sua veracidade.

\section{Referências}

1. Duque AM, Leal MCC, Marques AP0, Eskinazi FMV, Duque AM. Violência contra idosos no ambiente doméstico: prevalência e fatores associados (Recife/ PE). Cienc Saude Colet. 2012;17(8):2199-208. doi: https://doi.org/10.1590/S1413-81232012000800030.

2. Krug EG, Mercy JA, Dahlberg LL, Zwi AB. The world report on violence and health. Lancet.

2002;360 0ct 5;360(9339):1083-8. doi: https://
doi.org/10.1016/S0140-6736(02)11133-0.

3. Stöckl H, Penhale B. Intimate partner violence and its association with physical and mental health symptoms among older women in Germany. J Interpers Violence. 2015;30(17):3089-111. doi: https://doi.org/10.1177/0886260514554427. 
4. Stöckl H, March L, Pallitto C, Garcia-Moreno C. Intimate partner violence among adolescents and young women: prevalence and associated factors in nine countries: a cross-sectional study. BMC Public Health. 2014 Jul 25;14:751. doi: https://doi.org/10.1186/1471-2458-14-751.

5. Whitaker DJ, Haileyesus T, Swahn M, Saltzman LS. Differences in frequency of violence and reported injury between relationships with reciprocal and nonreciprocal intimate partner violence. Am J Public Health. 2007;97(5):941-7. doi: https://doi.org/10.2105/AJPH.2005.079020.

6. World Health Organization. Global and regional estimates of violence against women: prevalence and health effects of intimate partner violence and non-partner sexual violence. [Geneva]: WHO; 2013.

7. Desmarais SL, Reeves KA, Nicholls TL, Telford R0, Fiebert MS. Prevalence of physical violence in intimate relationships, part 1: rates of male and female victimization. Partner Abuse. 2012;3(2):1-6. doi: https://doi.org/10.1891/1946-6560.3.2.140.

8. Afifi TO, Henriksen CA, Asmundson GJG, Sareen $\mathrm{J}$. Victimization and perpetration of intimate partner violence and substance use disorders in a nationally representative sample. J Nerv Ment Dis. 2012;200(8):684-91. doi: https:// doi.org/10.1097/NMD.0b013e3182613f64.

9. Renner LM, Habib L, Stromquist AM, Peek-Asa C. The association of intimate partner violence and depressive symptoms in a cohort of rural couples. J Rural Health. 2014;30(1):50-8. doi: https://doi.org/10.1111/jrh.12026.

10. Burnes D, Pillemer K, Caccamise PL, Mason A, Henderson CR, Jr, Berman J, et al. Prevalence of and risk factors for elder abuse and neglect in the community: a population-based study. J Am Geriatr Soc. 2015;63(9):1906-12. doi: https://doi.org/10.1111/jgs.13601.

11. Bonomi AE, Anderson ML, Reid RJ, Carrell D, Fishman $\mathrm{PA}$, Rivara FP, et al. Intimate partner violence in older women. Gerontologist. 2007;47(1):34-41. doi: https://doi.org/10.1093/geront/47.1.34.

12. Guedes DT, Curcio CL, Llano BA, Zunzunegui MV, Guerra R. [The gender gap in domestic violence in older adults in Latin America: the IMIAS study]. Rev Panam Salud Publica. 2015;37(45):293-300. [Portuguese]. PMID: 26208199.

13. Bolsoni CC, Coelho EBS, Giehl MWC, d'Orsi E. Prevalência de violência contra idosos e fatores associados, estudo de base populacional em Florianópolis, SC. Rev Bras Geriatr Gerontol. 2016;19(4):671-82. doi: https://doi. org/10.1590/1809-98232016019.150184.

14. Warmling D, Lindner SR, Coelho EBS. Prevalência de violência por parceiro íntimo em idosos e fatores associados: revisão sistemática. Cien Saude Colet. 2017;22(9):3111-3125. doi: https://doi. org/10.1590/1413-81232017229.12312017.

15. Instituto Brasileiro de Geografia e Estatística. Estimativas populacionais residentes, em 1. de julho de 2009, segundo os municípios. Brasília, DF; 2009 [acesso 16 dez. 2020]. Disponível em: http:/www.ibge.gov.br/home/estatistica/ populacao/estimativa2009/P0P2009_D0U.pdf

16. Schneider IJC, Confortin SC, Bernardo CO, Carvalho CB, Antes DL, Pereira KG, et al. Estudo de coorte EpiFloripa Idoso: métodos, aspectos operacionais e estratégias de seguimento. Rev Saude Publica. 2017;51:104. doi: https://doi.org/10.11606/s1518-8787.2017051006776.

17. Hasselmann MH, Reichenheim ME. Adaptação transcultural da versão em português da Conflict Tactics Scales Form R (CTS-1), usada para aferir violência no casal: equivalências semântica e de mensuração. Cad. Saude Publica. 2003; 19(4): 1083-1093. https://doi. org/10.1590/S0102-311X2003000400030.

18. Folstein MF, Folstein SE, McHugh PR. "Minimental state": a practical method for grading the cognitive state of patients for clinician. J Psychiatr Res. 1975;12(3):189-98. doi: https:// doi.org/10.1016/0022-3956(75)90026-6.

19. Paradela EM, Lourenço RA, Veras RP. Validação da escala de depressão geriátrica em um ambulatório geral. Rev Saude Publica. 2005;39(6):918-23. doi: https://doi.org/10.1590/S0034-89102005000600008.

20. Rosa TEC, Benício MHA, Latorre MRD0, Ramos LR. Fatores determinantes da capacidade funcional entre idosos. Rev Saude Publica. 2003;37(1):40-8. doi: https://doi.org/10.1590/S0034-89102003000100008.

21. Rosay AB, Mulford CF. Prevalence estimates and correlates of elder abuse in the united states: the national intimate partner and sexual violence survey. J Elder Abuse Negl. 2017;29(1):1-14. doi: https://doi.org/10.1080/08946566.2016.1249817.

22. Hellemans S, Buysse A, De Smet 0, Wietzker A. Intimate partner violence in Belgium: prevalence, individual health outcomes, and relational 
correlates. Psychol Belg. 2014;54(1):79-

96. doi: https://doi.org/10.5334/pb.af.

23. Coker AL, Davis KE, Arias I, Desai S, Sanderson M, Brandt HM, et al. Physical and mental health effects of intimate partner violence for men and women. Am J Prev Med. 2002;23(4):260-8. doi: https://doi.org/10.1016/S0749-3797(02)00514-7.

24. Lindner SR, Coelho EBS, Bolsoni CC, Rojas PF, Boing AF. Prevalência de violência física por parceiro íntimo em homens e mulheres de Florianópolis, Santa Catarina, Brasil: estudo de base populacional. Cad Saude Publica. 2015;31(4):815-26. doi: http:// dx.doi.org/10.1590/0102-311X00159913.

25. Johnson MP. Conflict and control: gender symmetry and asymmetry in domestic violence. Violence Against Women. 2006;12(11):1003-18. doi: https://doi.org/10.1177/1077801206293328.

26. Próspero M. The effect of coercion on aggression and mental health among reciprocally violent

\section{Abstract}

objective: To verify prevalence of intimate partner violence among elderly people and to identify associated factors. Methods: This was a cross-sectional populationbased study using data from the EpiFloripa Idoso Study, with elderly people living in Florianópolis, Santa Catarina, Brazil, between 2013 and 2014. Prevalence rates were described, and factors associated using Poisson regression were analyzed, for violence suffered and perpetrated (committed by the elderly), stratified by sex. Results: Among 651 elderly people, 48.3\% of males and $46.4 \%$ of females suffered intimate partner violence. Psychological violence suffered (48.3\% and 44.8\%) and perpetrated (49.8\% and 44.5\%) predominated in males and females, respectively. Perpetrated violence was associated with poor/very poor self-perceived bealth $(P R=1.74)$ among males, and with regular selfperceived health $(P R=1.53)$ and separated/divorced marital status $(P R=1.86)$ among females. Conclusion: Symmetry was found between the sexes for prevalence of intimate partner violence, for all directionalities of violence analyzed.

Keywords: Intimate Partner Violence; Prevalence; Aged; Cross-Sectional Studies. couples. J Fam Violence. 2008;23(3):195-202. doi: https://doi.org/10.1007/s10896-007-9143-6.

27. Casimiro C. Violências na conjugalidade: a questão da simetria do género. Anal Soc. 2008:43(188):579-601.

28. Warmling D, Araújo CAH, Lindner SR, Coelho EBS. Qualidade de vida de mulheres e homens idosos em situação de violência por parceiro íntimo. Rev Bras Geriatr Gerontol. 2021;24(6):e200268. doi: https:// doi.org/10.1590/1981-22562020024.200268.

29. Plazaola-Castaño J, Ruiz-Perez I, EscribáAgüir V, Montero-Piñar I, Vives-Cases C. The assessment of intimate partner violence in Spanish women: the index of spouse abuse.J Interpers Violence. 2011;26(16):3267-88. doi https://doi.org/10.1177/0886260510393005.

30. Confortin SC, Schneider IJC, Antes DL, Cembranel F, Ono LM, Marques LP, et al. Condições de vida e saúde de idosos: resultados do estudo de coorte EpiFloripa Idoso. Epidemiol Serv Saude. 2017;26(2):305-17. doi: https://doi.org/10.5123/s1679-49742017000200008.

\section{Resumen}

Objetivo: Verificar la prevalencia de violencia por pareja intima en ancianos e identificar factores asociados. Métodos: Estudio poblacional transversal, del estudio EpiFloripa Idoso, con ancianos residentes en Florianópolis, Santa Catarina, Brasil, entre 2013 y 2014. Se describieron las prevalencias y se analizaron los factores asociados por la regresión de Poisson, para la violencia sufrida y perpetrada (cometida por los ancianos), estratificada por sexo. Resultados: Entre 651 ancianos, 48,3\% bombres y 46,4\% mujeres sufrieron violencia cometida por la pareja. La violencia psicológica sufrida (48,3\% y 44,8\%) y perpetrada (49,8\% y 44,5\%) predominó en hombres y mujeres, respectivamente. La violencia perpetrada se asoció con la autopercepción de mala/ muy mala salud $(R P=1,74)$ en los ancianos, autopercepción de salud regular $(R P=1,53)$ y el estado civil separado / divorciado $(R P=1,86)$ en las ancianas. Conclusión: Existió una simetría entre los sexos en la prevalencia de la violencia por pareja intima, en todas las direcciones analizadas.

Palabras clave: Violencia de Pareja; Prevalencia; Anciano; Estudios Transversales.

Recebido em 17/12/2020

Aprovado em 04/07/2021 